\title{
Two-dimensional unsteadiness map of oblique shock wave/boundary layer interaction with sidewalls
}

\author{
P. K. Rabey ${ }^{1} \dagger$, S. P. Jammy ${ }^{2}$ P. J. K. Bruce ${ }^{1}$, and N. D. Sandham ${ }^{3}$ \\ ${ }^{1}$ Department of Aeronautics, Imperial College London, London SW7 2AZ, UK \\ ${ }^{2}$ Department of Mechanical Engineering, School of Engineering and Applied sciences, SRM \\ University AP Amaraviti, India \\ ${ }^{3}$ Faculty of Engineering and the Environment, University of Southampton, Southampton SO17 \\ $1 \mathrm{BJ}, \mathrm{UK}$
}

(Received xx; revised xx; accepted $\mathrm{xx}$ )

The low-frequency unsteadiness of oblique shock wave/boundary layer interactions (SBLIs) has been investigated using large-eddy simulation (LES) and high-frequency pressure measurements from experiments. Particular attention has been paid to off-centreline behaviour: the LES dataset was generated including sidewalls and experimental pressure measurements were acquired across the entire span of the reflected shock foot. The datasets constitute the first maps of low-frequency unsteadiness in both streamwise and spanwise directions. The results reveal that significant low-frequency shock motion (with $S t \approx 0.03$ ) occurs away from the centreline, along most of the central separation shock and in the corner regions. The most powerful low frequency unsteadiness occurs offcentre, likely due to the separation shock being strengthened by shocks arising from the swept interactions on the sidewalls. Both simulation and experimental results exhibit asymmetry about the spanwise centre. In simulations, this may be attributed to a lack of statistical convergence; however, the fact that this is also seen in experiments is indicative that some SBLIs may exhibit some inherent asymmetry across the two spanwise halves of the separation bubble. There is also significant low-frequency power in the corner separations. The relation of the unsteadiness in the corner regions to that in the centre is investigated by means of two-point correlations: a key observation is that significant correlation does not extend across the attached flow channel between the central and corner separations.

Key words:

\section{Introduction}

SBLI unsteadiness is relevant for engine intake design. Despite this, off-centre unsteadiness has been largely neglected in experiments and the majority of simulations employ spanwise periodic boundary conditions (Garnier 2009; Pasquariello et al. 2017). In particular, researchers are interested in the structurally-fatiguing low-frequency reflected shock motion which occurs at Strouhal numbers around $S t=f L_{\mathrm{sep}} / U_{\infty}=0.03$ (Dupont et al. 2006). While much research has been conducted to further our understanding of

$\dagger$ Email address for correspondence: pr1010@ic.ac.uk 
two-dimensional SBLI unsteadiness, three-dimensional studies have been highlighted as an important area for future research (Dussauge et al. 2006; Clemens \& Narayanaswamy 2014). In particular it is not fully understood how the dynamic behaviour of the corner separations that exist in real geometries relates to phenomena observed at the centreline.

It has been established that corner separations affect the mean characteristics of the central separation. Bruce et al. (2011) showed that corner separations influence the extent of the main separation region of normal SBLIs; this was expanded upon by Babinsky et al. (2013) who proposed that interactions can be classified as 'quasi-two-dimensional' or with increased or decreased separation based on the viscous aspect ratio $\delta / w$ of the configuration (where $\delta$ is the incoming boundary layer height and $w$ is the working section width). However the exact relationship between the two is complex; Benek et al. (2014), Benek et al. (2016) and Poggie \& Porter (2018) conducted LES of test cases with varying viscous aspect ratios without successfully finding a flow parameter which collapsed the trends for the observed variation in separation across test cases with different shock strengths and incoming Mach numbers.

Wang et al. (2015) subsequently proposed that the two-dimensionality of oblique SBLIs depends on the conical growth from the shock generator leading edge of the swept fin interactions between the shock generator, incident shock and sidewall boundary layers. These interactions affect the main SBLI by distorting the incident shock. This further shows that interactions are strongly influenced by the sidewalls, as well as the height of the domain.

There has been previous speculation on links between corner and central separation region unsteadiness. An early suggestion of a relation arose from the particle image velocimetry (PIV) measurements on an oblique SBLI summarised by Dupont et al. (2005) and Dussauge et al. (2006). The PIV in the $x-z$ plane parallel to the wall revealed the presence of two counter-rotating 'tornado' vortices behind the separation shock extending to $y \sim 0.5 \delta$. From the velocity gradients in the $x-z$ plane, they estimated that the angular frequency of the vortices was of the same order as the low-frequency shock unsteadiness. They concluded that further work was required to determine what effect the sidewalls and wind tunnel aspect ratios have on this phenomenon. Oil flow visualisations by Babinsky et al. (2013) revealed that the foci of such vortices move inwards when blockage is placed in the corners and those of Grossman \& Bruce (2018) show the same tendency for increasing duct aspect ratio $R=w / h$, where $h$ is the height of the leading edge of the shock generator from the tunnel floor.

This link was then investigated by Garnier (2009) in an SBLI simulation with sidewalls. Garnier (2009) investigated the correlation between the reflected shock motion and the corner flows and central flow counter-rotating vortices but found no link; however due to the short signal time of the simulation, motions with frequencies lower than $200 \mathrm{~Hz}$ could not be investigated. Historically, investigation of low-frequency dynamics via numerical simulations is lacking due to the challenges of obtaining a sufficiently long run time (Pasquariello et al. 2017), which are increased further by the inclusion of sidewalls. Experiments have an advantage in this sense if longer run times are available; however it is difficult to obtain high resolution spatial data over a sizeable extent.

Funderburk \& Narayanaswamy (2016) experimentally investigated this link by taking pressure measurements under the central and corner separations of a compression ramp SBLI. Noting the differences in the cross-coherences of the power spectra under the most energetic locations at the centre and corner with locations upstream and downstream of them, they concluded that the separations behave independently of each other. However they were not able to simultaneously measure the pressure in the two regions due to the constraints of the experimental arrangement. 


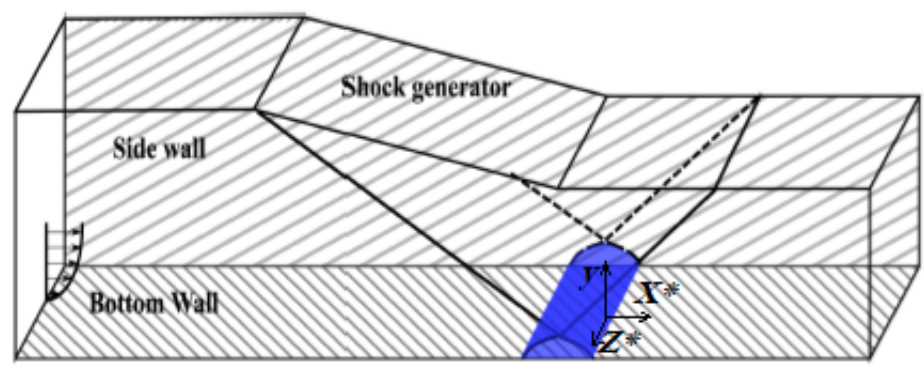

Figure 1: Schematic diagram of numerical domain.

Therefore by utilising both LES and experimental data, the purpose of this paper is two-fold: to map the unsteadiness across the span of oblique SBLIs and to evaluate the influence of off-centre unsteadiness on the well-documented low-frequency motions at the spanwise centre. Data are taken from two independent studies with similar interaction strengths, but with different Mach numbers and aspect ratios. Our interest is in the extent to which low-frequency phenomena are similar.

\section{Methodology}

\subsection{Numerical approach - "Test case 1"}

The numerical dataset is taken from the full-span LES of a $9^{\circ}$ oblique SBLI at Mach 2.7 and $R=2(\delta / w=0.069)$ by Wang et al. (2015). Previous LES (Wang et al. 2015) showed that the corner and its neighbouring attached flow region are not significantly changed by aspect ratios in the range 1 to 4 . This configuration generates an expected inviscid incident shock strength of $p_{2} / p_{1}=1.82$. The Reynolds number based on the boundary layer compressible momentum thickness at the shock impingement location was $R e_{\theta}=4300$ and the boundary layer compressible displacement thickness was $\delta^{*}=2.3 \mathrm{~mm}$. The simulated domain was the entire channel including the shock generator and both sidewalls, as shown in figure 1 . The streamwise $\times$ wall normal $\times$ spanwise resolution of the grid was $480 \times 416 \times 758$. Full details including a description of the grid sensitivity study are provided in Wang et al. (2015).

In the present work the WH2 $(R=2)$ simulation of Wang et al. $(2015)$ was continued for approximately 23 periods of the expected low-frequency motion based on $S t=0.03$ $\left(f_{0}=629 \mathrm{~Hz}, T_{0}=1.59 \mathrm{~ms}\right)$, following a transient time of about 4 periods. The pressure history on the bottom wall (see figure 1) was sampled every 20 simulation time-steps from which the power spectral densities (PSDs) of the signals were then computed using Welch's method with a $50 \%$ overlap. The PSDs were reconstructed with 2 segments at the lowest frequency and 32 at the high frequencies.

\subsection{Experimental set-up - "Test case 2"}

Experiments were conducted in the Imperial College supersonic wind tunnel to study the oblique SBLI formed by a $12^{\circ}$ wedge in a Mach 2 freestream at $R=1(\delta / w=0.036)$. The inviscid pressure rise across this incident shock is expected to be $p_{2} / p_{1}=1.88$ and the upstream boundary layer had $R e_{\theta} \approx 10000$ and $\delta^{*} \approx 1.2 \mathrm{~mm}$. Further details can be found in Grossman \& Bruce (2018).

The surface oil flow visualisation technique described in Grossman \& Bruce (2018) was employed to characterise the separation region at the floor. The pressure history on 


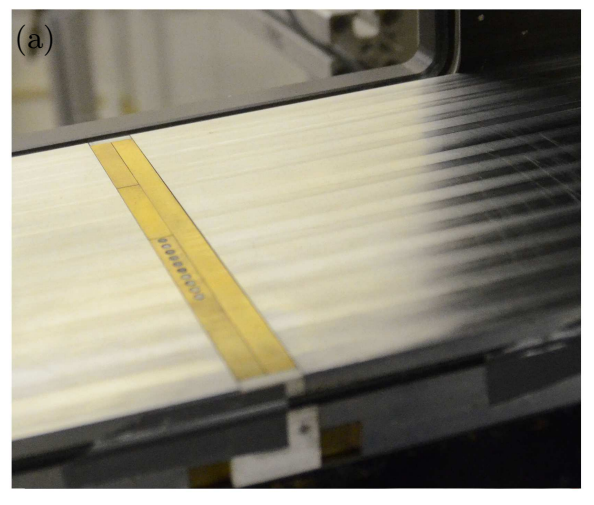

Figure 2: (a) Photograph and (b) CAD model showing experimental set-up.

the floor was recorded using high-frequency response Kulite standard version miniature pressure transducers XCQ-093-15PSIA, all $2.4 \mathrm{~mm}$ in diameter. Channels were sampled at $1 \mathrm{MHz}$ for $14 \mathrm{~s}$ during the steady state of the tunnel operation, equivalent to $6300 T_{0}$ where $T_{0}=2.22 \mathrm{~ms}\left(f_{0}=450 \mathrm{~Hz}\right)$ is one expected low-frequency period based on $S t=0.03$. Although not shown for brevity, convergence was confirmed as the same trends were found using one tenth of the available time series (still 630 low-frequency cycles).

The signals were obtained over a $10 \times 40$ streamwise-by-spanwise grid covering the central $94 \%$ of the working section width $w=150 \mathrm{~mm}$ with resolution $1.36 \mathrm{~mm} \times 3.6 \mathrm{~mm}$ or $0.04 L_{\text {sep }} \times 0.02 w$ where $L_{\text {sep }}$ is the centreline separation length. The PSDs were then computed using Welch's method with 1700 windows of length 16.4 ms with $50 \%$ overlap, giving a frequency resolution of $61 \mathrm{~Hz}$ or $S t=0.004$.

Ten sensors were mounted in a floor plug at the same streamwise position with a spanwise centre-to-centre spacing of $3.6 \mathrm{~mm}$, as shown in figure 2 . The floor plug could be placed at four locations across the working section span. A streamwise resolution of $1.4 \mathrm{~mm}$ was achieved by shifting the shock generator up and down with two streamwise plug locations. The shock generator was traversed vertically in increments of $1.21 \mathrm{~mm}$ in order to effectively shift the interactions by $\pm 1.36 \mathrm{~mm}$ in the streamwise direction. In shifting the shock generator by such small vertical increments, the aspect ratio was always within $R=1.02 \pm 0.017$ which caused minimal change in the confinement and in boundary layer thickness at the shock impingement.

\section{3. $S t=0.03$}

For the remainder of the paper the distribution in power contained in $S t=0.03$ will be considered. The choice in using $S t=0.03$ based on the centreline separation length is mostly due to historical convention in that this frequency is expected to be characteristic of the unsteadiness of a separated SBLI. However, it was also found that this was a good representation of the variation in energy contained in low frequencies across the span of the interactions and choices of other Strouhal numbers yielded similar trends. Example PSDs across the span of an interaction are shown in figure 3 from which it can be seen that the power at $S t=0.03$ captures the variation in low-frequency energy content at each location. 


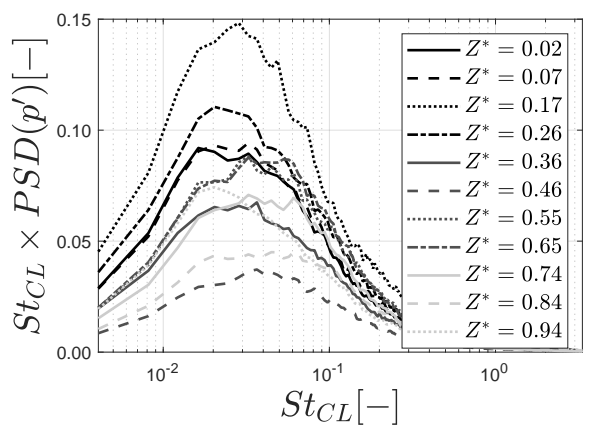

Figure 3: Example PSDs along $X^{*}=-1.30$ for test case 2 .

\section{Results}

\subsection{Mean separation regions}

Figures 4(a) and 4(b) show schlieren images of the numerical and experimental flowfields (in the $x-y$ plane). Figures $4(\mathrm{c})$ and $4(\mathrm{~d})$ show the mean separation regions in the $x-z$ plane: for the numerical case, this is shown by a white line along which $(\partial u / \partial y)_{w}=0$; while in the experiment, the oil collects at lines for which the shear stress is zero and therefore the region for $(\partial u / \partial y)_{w} \leqslant 0$ is apparent within the thick white lines in the photograph. As expected, the position of the separation line inferred from the oil flow in 4(d) aligns with the foot of the separation shock wave deduced from the schlieren in figure 4(b), consistent with previous reported results for incident-reflected SBLIs (Grossman \& Bruce 2018). $X^{*}$ is normalised by $L_{\text {sep }}$ and $Z^{*}$ by $w / 2$.

A similar centreline separation length is caused by the interactions in both cases ( $0.29 w$ in the LES compared to $0.23 w$ in the experiment). A notable difference between the separation regions is that, while the LES separation line is straight, there is a large meandering in the experimental case in the positive $z$-plane $O\left(0.3 L_{\mathrm{sep}}\right)$. This is attributed to an imperfection in the wind tunnel contraction or nozzle giving rise to different boundary layer characteristics across the span. Test case 1 exhibits a typical 'medium viscous aspect ratio' separation shape and test case 2 shows a 'large viscous aspect ratio' shape as proposed by Babinsky et al. (2013).

The foci of the tornado vortices described in $\S 1$ are marked in figures $4(\mathrm{c})$ and $4(\mathrm{~d})$. In test case 1 these are at around $36 \%$ of the half-span from the walls whereas in test case 2 these are at about $24 \%$ of the half-span. Both Grossman \& Bruce (2018) and Wang et al. (2015) show a slight increase in distance from the wall to the foci for increasing $R$ so this difference might be attributed to the difference in aspect ratio between the test cases.

\subsection{Wall pressure spectra}

Figures 4(e) and 4(f) show the energy content in the weighted power spectral density (WPSD) at $S t=0.03$ on the floor plane of the interactions. This is made dimensionless by integrating over the Strouhal number range. Figures $4(\mathrm{e})$ and $4(\mathrm{f})$ highlight that high-energy low-frequency motions exist across the span of the central separation regions. There is also some low-frequency motion near the sidewalls in both cases. In the simulations this peaks at approximately $0.7 L_{\text {sep }}$ downstream of the peak unsteadiness of the central separation, outside the range of experimental measurements. However, 
(a)

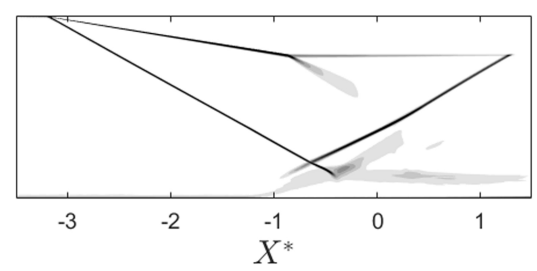

(c)

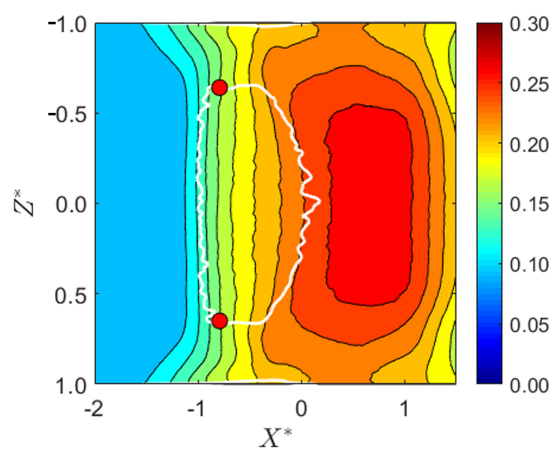

(e)

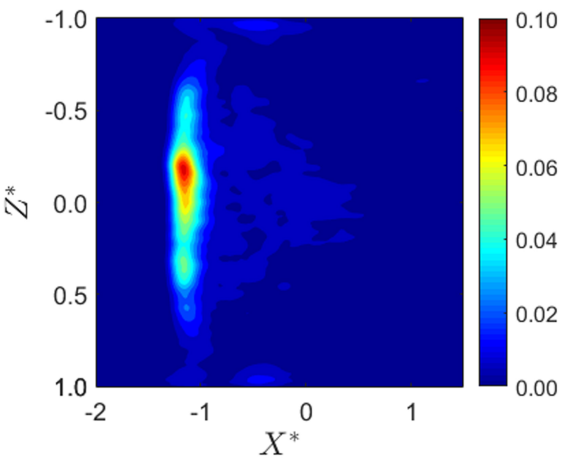

(b)

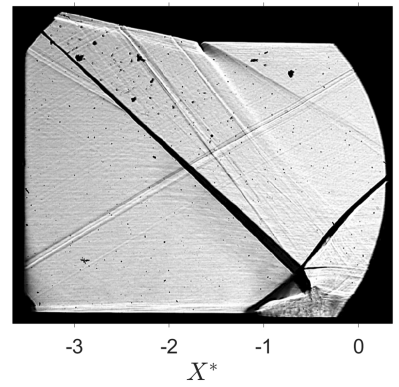

(d)

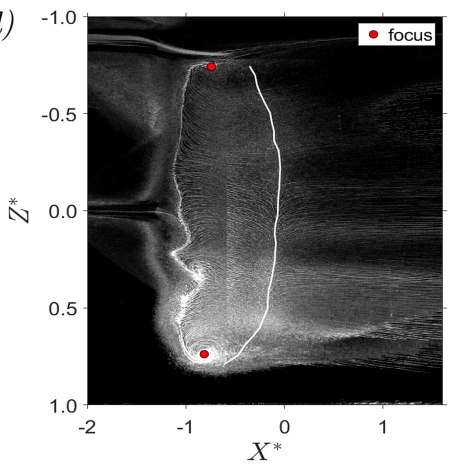

(f)

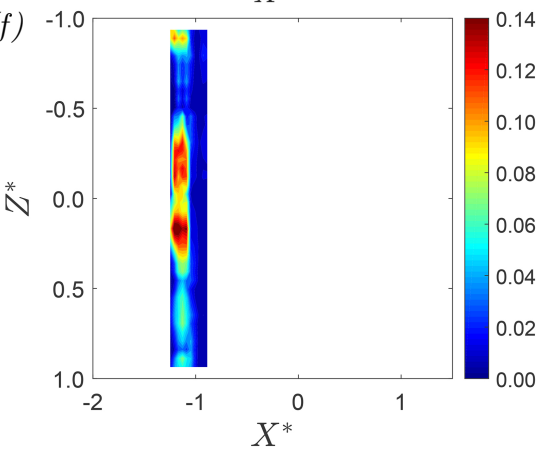

Figure 4: Schlieren images for (a) test case 1 and (b) test case 2. Mean separation regions on floor plane with (c) contours of wall pressure for test case 1 and (d) artificially emphasised reattachment line for test case 2. Map of WPSDs at $S t=0.03$ on bottom wall plane for (e) test case 1 and (f) test case 2 .

experimental results do show energy comparable to that seen at the centreline in the same streamwise location as at the centre. Meanwhile, there is little low-frequency energy in the attached channels between the central and corner separations, which might have been expected as separation is a condition of low-frequency SBLI unsteadiness (Babinsky \& Harvey 2011).

The higher energy in the corner separations of test case 2 might be due to the absolute height of the duct being three times larger than that of test case 1, which might result in larger interactions of the sidewall boundary layers with the incident shock. This hypothesis is supported by the apparent thickening of the incident shock towards the floor seen in the spanwise-integrated schlieren image for test case 2 in figure 4(b). This 
(a)

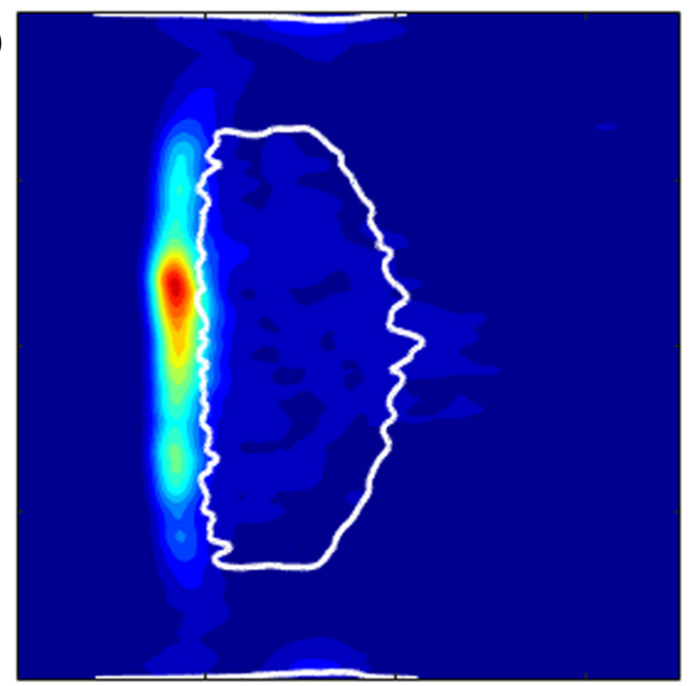

(b)

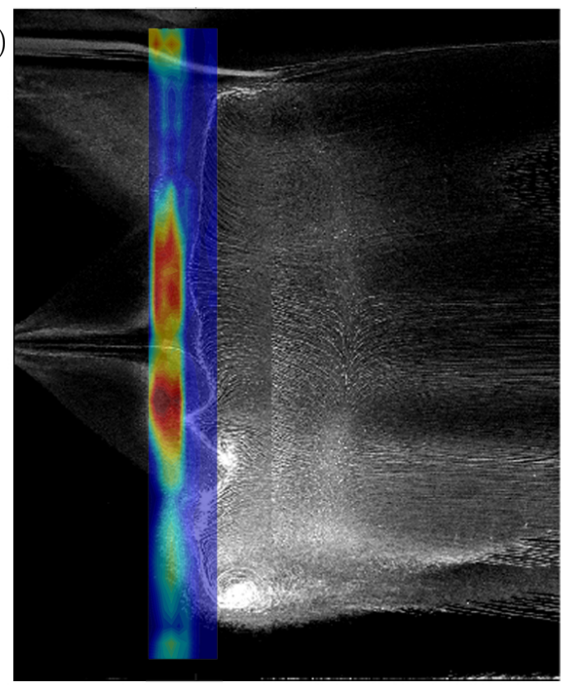

Figure 5: WPSD map superimposed with mean separation region for (a) test case 1 and (b) test case 2 .

is because these interactions are equivalent to a swept SBLI (Wang et al. 2015). Hence more energetic low-frequency motion might be expected due to the larger separations.

Comparison of figures $4(\mathrm{~d})$ and $4(\mathrm{f})$ for test case 2 reveal that regions of high lowfrequency power in the central separation region are correlated with locations where the separation line (inferred from oil flow) is locally displaced upstream. This is further explored in figure 5 where the WPSD maps have been superimposed onto the separation regions. Figure 5(b) confirms that, for test case 2 (with $A=1$ ), regions of the interaction where the separation line is more upstream do exhibit more low-frequency power. This trend is less obvious in figure $5(\mathrm{a})$, possibly because the higher $R$ of test case $1(R=2)$ gives rise to less significant span-wise variations in the location of the separation line.

The spanwise power distribution at the streamwise lines containing the point with the highest energy $\left(X^{*}=-1.14\right.$ in test case 1 and $X^{*}=-1.30$ in test case 2$)$ are presented in figure 6 . It shows that the most energetic instances of the low frequencies do not occur at the centreline. This case falls into category (ii) from Wang et al. (2015) in which the sidewall effects have reached the central region of the flow, resulting in significant spanwise variations in the incident shock strength.

Off-centre peaks appear in all the cases in figure 6 . In all the cases except the numerical case at $S t=0.03$ (which is most affected by the sampling time), there are two offcentre peaks. In the experiment these are not quite symmetric about the centreline. However the ratio of the power across the peaks for the different Strouhal numbers remains fairly constant (at around 1.2), implying convergence. It is therefore possible that some interactions exhibit a more prevalent low-frequency behaviour on one side of the spanwise centre.

\subsection{Two-point correlations}

Given the significant low-frequency energy content near the sidewalls, the influence of pressure fluctuations near the wall on those at the centre was examined by considering the two-point correlations $R_{p p}$ of the pressure signals. This is defined as in equation 3.1, where $p^{\prime}(z, \tau)$ is the pressure fluctuation at a location $z$ and time instance $\tau$ and $p\left(z+r_{2}, \tau^{\prime}\right)$ 

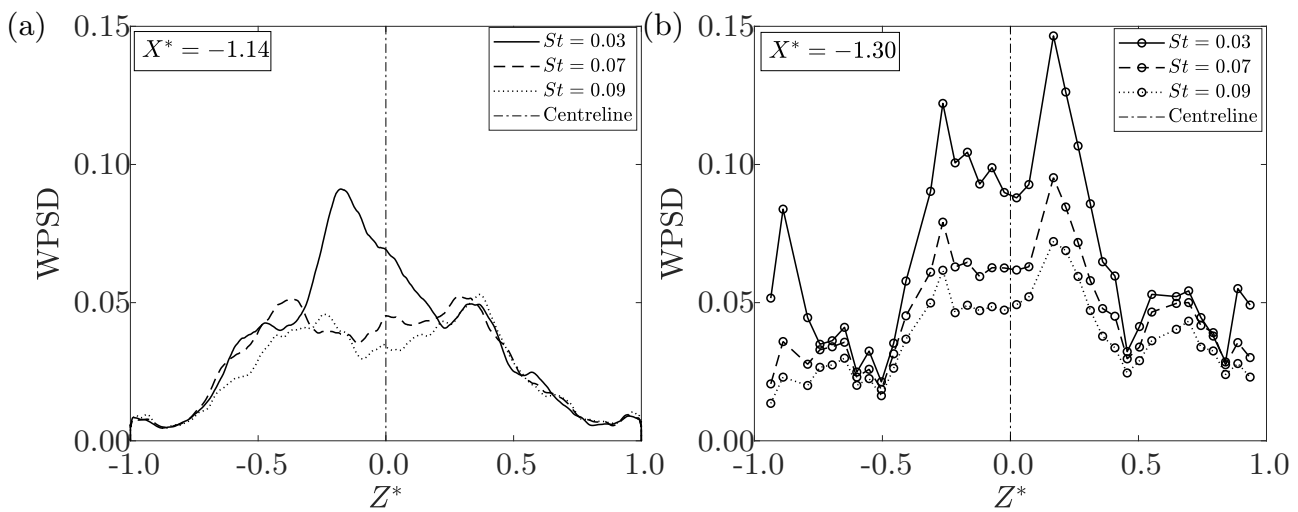

Figure 6: WPSD at streamwise locations of highest $S t=0.03$ energy content for (a) test case 1 and (b) test case 2 .
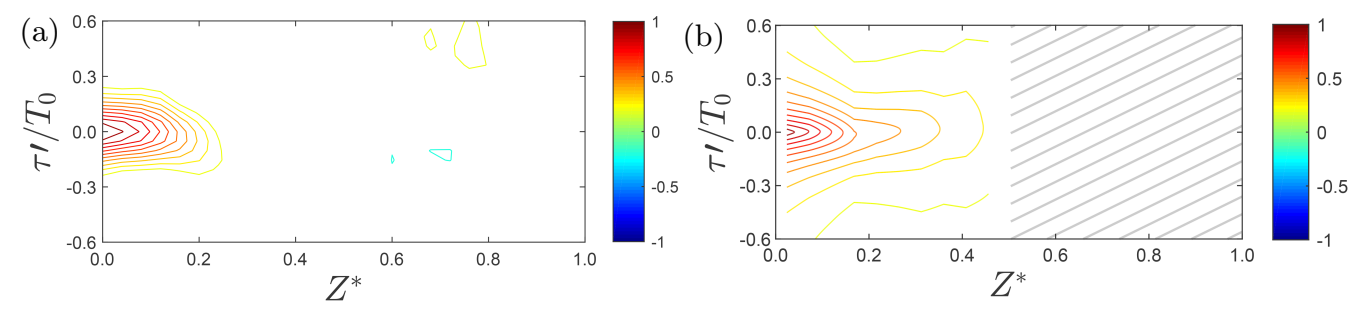

Figure 7: Two-point correlations from centreline pressure at (a) $Z^{*}=0.00$ for test case 1 and (b) $Z^{*}=0.02$ for test case 2 along same spanwise axis.

is the pressure fluctuation at a location separated from $z$ by $r_{2}$ in the $z$-direction and at a time delay $\tau^{\prime}$. A positive time delay corresponds to the event at time $\tau$ occurring first. The signals are first low-pass filtered with a cut-off frequency of $S t=0.15$ to focus on low-frequency behaviour. Contour levels $-0.2<R_{p p}<0.2$ are suppressed to mitigate noise.

$$
R_{p p}\left(z ; r_{2}, \tau^{\prime}\right)=\frac{\left\langle p^{\prime}(z, \tau) p^{\prime}\left(z+r_{2}, \tau^{\prime}\right)\right\rangle}{p_{R M S}(z) p_{R M S}\left(z+r_{2}\right)}
$$

Figure 7 shows that significant correlations of centreline behaviour diminish beyond about $20 \%$ of the half-span. In contrast, figure 8 shows that there are significant correlations from the near-wall point into the central flow until the spanwise location of the vortex foci shown in figures $4(\mathrm{c})$ and $4(\mathrm{~d})$ (at $Z^{*}=0.64$ and $Z^{*}=0.76$ in test cases 1 and 2 respectively). Therefore, along the same streamwise plane, there is not a significant relation of the pressure between the two separation regions.

The influence of the corner separation unsteadiness from a different streamwise plane on the central region was evaluated, for which only the numerical data could be employed. A further two-point correlation was performed between the signal at $X^{*}=-0.41$, $Z^{*}=0.96$ (the high energy peak in the corner separation of figure $4(\mathrm{e})$ ) and across the span of the domain at $X^{*}=-1.14$. This is shown in figure 9 which highlights a negative correlation with the pressure signal $0.74 L_{\text {sep }}$ upstream, at a slightly later time. However, 

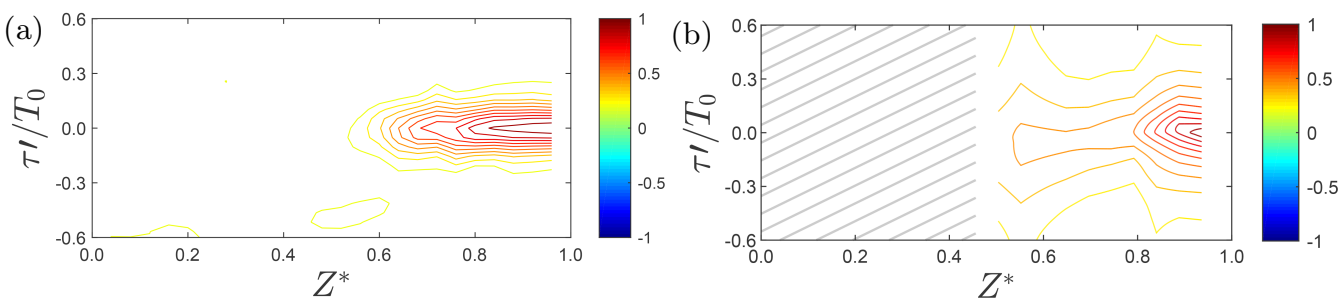

Figure 8: Two-point correlations from near wall pressure at (a) $Z^{*}=0.96$ for test case 1 and (b) $Z^{*}=0.94$ for test case 2 along same spanwise axis.

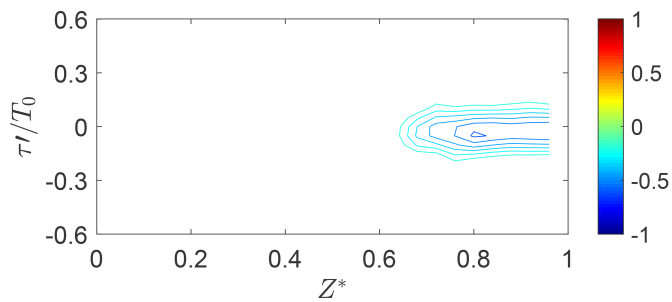

Figure 9: Two-point correlations from near wall pressure at $Z^{*}=0.96, X^{*}=-0.41$ and spanwise points along $X^{*}=-1.14$.

this correlation extends less far into the central flow and therefore does not appear to be affecting the central unsteadiness.

None of the surface pressure correlations, consistently between the experiments and simulations, show any indication of a low-frequency unsteady connection between the sidewall and central separations. This is in agreement with the conclusion drawn from separate central and corner separation measurements by Funderburk \& Narayanaswamy (2016). Due to the datasets only being available at the floor, it is not possible to directly assess the correlation of the two regions above the bottom wall on different wall-normal planes. This could potentially occur via the interaction of the tornado vortices and sidewall vortices.

\section{Conclusion}

Two oblique SBLIs of similar strength have been investigated. Both exhibit the most powerful instances of low-frequency motions off-centre, likely due to the incident shocks being locally strengthened by their interaction with the sidewall boundary layers. Both showed asymmetric unsteadiness behaviour, the reasons for which appear to be different. The experimental separation region exhibited significant asymmetry, which correlated with a varying spanwise power in the low-frequency motions. An asymmetry was also seen in the spanwise distribution of power in $S t=0.03$ in the LES data; however this may be an artefact of the data only comprising 23 expected low-frequency periods. At frequencies for which there are more available cycles and hence better convergence $(S t=0.07$ and 0.09), the unsteadiness was more symmetric with peaks away from the centre.

Nonetheless, the results show that conventional assumptions of two-dimensionality at the centre of an interaction may be invalid even at moderate aspect ratios. This could explain some discrepancies between spanwise-periodic simulations and experiments as the sidewalls affected the mean unsteady characteristics, even though the cross- 
correlations at low frequency showed no direct dynamical link between the central and corner separations.

The low-frequency energy content was more prevalent in the corner separations in the experimental case. This could be attributed to the smaller test section aspect ratio giving the swept sidewall SBLIs greater influence, although more tests at different aspect ratios are desirable in order to verify this. While the two-point correlations of the pressure at the near-sidewall points with the pressure at the more central points were insignificant, these datasets were limited to the pressure history at the floor. Therefore, the study cannot completely rule out the possibility of the corner separations affecting the central unsteadiness. It would be of interest to compare the results to studies with 'quasi-twodimensional' and highly three-dimensional interaction regions which may reveal different relations between central and corner separations.

\section{Acknowledgements}

The authors would like to thank UK Turbulence Consortium under EPSRC grant $\mathrm{EP} / \mathrm{L} 000261 / 1$ for the computing time.

\section{REFERENCES}

Babinsky, H. \& Harvey, J. K. 2011 Shock Wave-Boundary-Layer Interactions. Cambridge: Cambridge University Press.

Babinsky, H., Oorebeek, J. \& Cottingham, T. G. 2013 Corner effects in reflecting oblique shock-wave/boundary-layer interactions. In 51st AIAA Aerospace Sciences Meeting including the New Horizons Forum and Aerospace Exposition, pp. 1-10.

Benek, J. A., Suchyta, C. J. \& Babinsky, H. 2014 The Effect of Wind Tunnel Size and Shock Strength on Incident Shock Boundary Layer Interaction Experiments. AIAA Aviation Forum (June), 1-30.

Benek, J. A., Suchyta, C. J. \& Babinsky, H. 2016 Simulations of Incident Shock Boundary Layer Interactions. In 54th AIAA Aerospace Sciences Meeting.

Bruce, P. J. K., Burton, D. M. F., Titchener, N. A. \& Babinsky, H. 2011 Corner effect and separation in transonic channel flows. Journal of Fluid Mechanics 679, 247-262.

Clemens, N. T. \& Narayanaswamy, V. 2014 Low-Frequency Unsteadiness of Shock Wave/Turbulent Boundary Layer Interactions. Annual Review of Fluid Mechanics 46 (September), 469-492.

Dupont, P., Haddad, C., Ardissone, J. P. \& Debiève, J.-F. 2005 Space and time organisation of a shock wave/turbulent boundary layer interaction. Aerospace Science and Technology 9 (7), 561-572.

Dupont, P., Haddad, C. \& Debiève, J.-F. 2006 Space and time organization in a shockinduced separated boundary layer. Journal of Fluid Mechanics 559, 255.

Dussauge, J.-P., Dupont, P. \& Debiève, J.-F. 2006 Unsteadiness in shock wave boundary layer interactions with separation. Aerospace Science and Technology 10, 85-91.

Funderburk, M. \& NARAyAnaswamy, V. 2016 Experimental investigation of primary and corner shock boundary layer interactions at mild back pressure ratios. Physics of Fluids 28 (8).

Garnier, E. 2009 Stimulated Detached Eddy Simulation of three-dimensional shock/boundary layer interaction. Shock Waves 19 (6), 479-486.

Grossman, I. J. \& Bruce, P. J. K. 2018 Confinement effects on regular-irregular transition in shock-wave-boundary-layer interactions. Journal of Fluid Mechanics 853, 171-204.

Pasquariello, V., Hickel, S. \& Adams, N. A. 2017 Unsteady effects of strong shock-wave / boundary-layer interaction at high Reynolds number. Journal of Fluid Mechanics 823, 617-657.

Poggie, J. \& Porter, K. M. 2018 Numerical Simulation of Sidewall Influence on Supersonic Compression Ramp Interactions. In 2018 Fluid Dynamics Conference, pp. 1-9. 
Wang, B., Sandham, N. D., Hu, Z. \& Liu, W. 2015 Numerical study of oblique shockwave/boundary-layer interaction considering sidewall effects. Journal of Fluid Mechanics $\mathbf{7 6 7}$ (2015), 526-561. 